\title{
Interacting Electrons and Holes in Quasi-2D Quantum Dots in Strong Magnetic Fields
}

\author{
P. HaWrylak ${ }^{a}$, W. Sheng ${ }^{a}$ And S.-J. Cheng ${ }^{b}$ \\ ${ }^{a}$ Institute for Microstructural Sciences, National Research Council of Canada \\ Ottawa, Ontario K1A OR6, Canada \\ ${ }^{b}$ Electrophysics Department, National Chiao Tung University \\ Hsinchu 30050, Taiwan, Republic of China
}

Theory of optical properties of interacting electrons and holes in quasi$-2 \mathrm{D}$ quantum dots in strong magnetic fields is discussed. In two dimensions and the lowest Landau level, hidden symmetries control the interaction of the interacting system with light. By confining electrons and holes into quantum dots hidden symmetries can be removed and the excitation spectrum of electrons and excitons can be observed. We discuss a theory electronic and of excitonic quantum Hall droplets at a filling factor $\nu=2$. For an excitonic quantum Hall droplet the characteristic emission spectra are predicted to be related to the total spin of electron and hole configurations. For the electronic droplet the excitation spectrum of the droplet can be mapped out by measuring the emission for increasing number of electrons.

PACS numbers: 73.21.-b, 83.35.-p, 73.21.La, 71.35.Ji, 78.67.Hc

\section{Introduction}

Optical properties of interacting electrons and holes are controlled by the electron hole Hamiltonian $H$, by the interband polarization operator $P$, and by the commutation relation of $P$ and $H[1,2]$. In strong magnetic fields and for spin polarized electrons, the analogy between electrons and holes and spin up and down electrons can be drawn. The polarization operator plays the role of spin raising operator and its spectrum is independent of Coulomb interactions. Hence it was believed that hidden symmetries prevent the optical detection of electronic excitations in a strongly interacting two-dimensional electron gas (2DEG) in strong magnetic fields. This belief was somehow mitigated by the discovery of bound 
"charged excitons" in strong magnetic fields [3], and the development of the understanding of these charged excitons in emission from the 2DEG [4-7]. To gain access to optically detected properties of the electronic system, the breaking of hidden symmetries was introduced by increasing the separation of holes and electrons [8], by localizing holes on acceptors [9], by introducing impurities into the electron gas [10], and by laterally confining the electron gas into quantum dots [11]. Recent experiments [12-14] show that it is now possible to fabricate high quality self-assembled quantum dots confining both electrons and holes and to study their optical properties in a strong magnetic field [14], i.e. in the quantum Hall regime. Our previous work focused on the evolution of emission and absorption spectra as a function of the number of electrons $\mathrm{Ne}$ and magnetic field. This included charged excitons, optical observation of Hund's rules, emission from high filled shells, and optical detection of magnetic field induced charge reconstruction in the spin polarized electron droplet [15-18]. In parallel, a theory for equal number of electrons and holes in optically excited self-assembled quantum dots was developed [19]. This theory drew on the analogies with the quantum Hall effect and showed that the hidden symmetries replace Hund's rules in partially filled shells of electron-hole dots. These symmetries can be destroyed and re-introduced by the application of strong magnetic field. A theory of excitonic artificial atoms in magnetic field was described recently in Ref. [20]. Here we briefly discuss hidden symmetries, in particular the symmetry associated with bi-excitons and spin, and its manipulation by magnetic field induced degeneracies [2, 20]. We next move to the regime of high magnetic field where the confining potential of the quantum dot removes the degeneracy of the lowest Landau level orbitals. The removal of degeneracy is critical for the destruction of hidden symmetries. We show that once the hidden symmetry is destroyed, optical spectra can be directly related to the excitation spectrum of excitonic and electronic droplets.

\section{Hidden symmetries}

In self-assembled quantum dots there is a finite number of bound states separated from the continuum. This number and their orbital character can be tuned by the magnetic field. The Fock-Darwin spectrum of two harmonic oscillators " $m$ " and " $n$ " is a good example. Let us assign a composite index $j=[m, n]$ to each state (we omit spin degrees of freedom $\sigma$ for simplicity). The general Hamiltonian of the interacting electron-hole system may be written in a compact form as:

$$
\begin{aligned}
H= & \sum_{i} E_{i}^{\mathrm{e}} c_{i}^{+} c_{i}+\sum_{i} E_{i}^{\mathrm{h}} h_{i}^{+} h_{i}-\sum_{i j k l}\left\langle i j\left|V_{\mathrm{eh}}\right| k l\right\rangle c_{i}^{+} h_{j}^{+} h_{k} c_{l} \\
& +\frac{1}{2} \sum_{i j k l}\left\langle i j\left|V_{\mathrm{ee}}\right| k l\right\rangle c_{i}^{+} c_{j}^{+} c_{k} c_{l}+\frac{1}{2} \sum_{i j k l}\left\langle i j\left|V_{\mathrm{hh}}\right| k l\right\rangle h_{i}^{+} h_{j}^{+} h_{k} h_{l} .
\end{aligned}
$$

The operators $c_{i}^{+}\left(c_{i}\right), h_{i}^{+}\left(h_{i}\right)$ create(annihilate) the electron or valence band hole 
in the state $|i\rangle$ with the single-particle energy $E_{i}$. The two-body Coulomb matrix elements are $\langle i j|V| k l\rangle$ for electron-electron (ee), hole-hole (hh) and electron-hole (eh) scattering, respectively.

The interaction of the system with light is described by the set of interband polarization operators $P^{+}\left(P^{-}\right)$which create(annihilate) electron-hole pairs $P^{+}=\sum_{i} c_{i}^{+} c_{i}\left(P^{-}=\sum_{i} c_{i}^{+}\right)$by annihilating (creating) photons. The polarization operator involves only one particle of a given type, i.e., one electron and one hole. The equation of motion for the polarization operator couples to many charge and spin operators through the commutator [18]

$$
\begin{aligned}
& {\left[H, P^{+}\right]=\sum_{i}\left(E_{i}^{\mathrm{e}}+E_{i}^{\mathrm{h}}\right) c_{i}^{+} h_{i}^{+}-\sum_{i j k l}\left\langle i j\left|V_{\mathrm{eh}}\right| k l\right\rangle c_{i}^{+} h_{j}^{+}} \\
& +\frac{1}{2} \sum_{i j k l}\left(\left\langle i j\left|V_{\mathrm{ee}}\right| k l\right\rangle-\left\langle i k\left|V_{\mathrm{eh}}\right| j l\right\rangle\right)\left(c_{i}^{+} h_{l}^{+} c_{j}^{+} c_{k}-c_{i}^{+} h_{k}^{+} c_{j}^{+} c_{l}\right) \\
& +\frac{1}{2} \sum_{i j k l}\left(\left\langle i j\left|V_{\mathrm{hh}}\right| k l\right\rangle-\left\langle i k\left|V_{\mathrm{eh}}\right| j l\right\rangle\right)\left(c_{l}^{+} h_{i}^{+} h_{j}^{+} h_{k}-c_{k}^{+} h_{i}^{+} h_{j}^{+} h_{l}\right) .
\end{aligned}
$$

The dynamics of the interband polarization requires the knowledge of both two-particle and four-particle operators, the dynamics of which has to be sought and truncated at some level of approximation. This coupling to many degrees of freedom is responsible for fast dephasing in semiconductors. To avoid this one must design our energy levels and matrix elements. If the single particle energy levels are degenerate, i.e. $E_{i}^{\mathrm{e}}+E_{i}^{\mathrm{h}}=E^{\mathrm{e}}+E^{\mathrm{h}}$ and the Coulomb matrix elements $\left\langle i j\left|V_{\text {eh }}\right| k k\right\rangle$ satisfy $\left\langle i j\left|V_{\text {eh }}\right| k k\right\rangle=\delta_{i j} V_{0}$, the first two terms $\sum_{i}\left(E_{i}^{\mathrm{e}}+E_{i}^{\mathrm{h}}\right) c_{i}^{+} h_{i}^{+} \pm \sum_{i j k l}\left\langle i j\left|V_{\mathrm{eh}}\right| k l\right\rangle c_{i}^{+} h_{j}^{+}=E_{X} P^{+}$turn out to be proportional to the polarization $P^{+}$. We are still left with the four-body terms. Fortunately, the four-body operators are proportional to the difference of matrix elements, e.g. $\left\langle i j\left|V_{\text {ee }}\right| k l\right\rangle-\left\langle i k\left|V_{\text {eh }}\right| j l\right\rangle$. Hence, if this difference can be made very small, the four-body terms vanish and $\left[H, P^{+}\right]=E_{X} P^{+}$. The degeneracy of single particle levels and the symmetry of (ee), (hh), and (eh) interactions cause a remarkable cancellation of the four-particle contribution and lead to a very simple dynamics of the interband polarization operator operating on a degenerate shell. The exact eigenstates coherent are constructed by a successive application of $P^{+}$, e.g. $|N\rangle=\left(P^{+}\right)^{N}|v\rangle$. The energy of these multiplicative states depends linearly on the number of excitons $N$. Hence the energy of addition/subtraction of excitons from these states does not depend on the number of excitons $N$. This is the essence of the "hidden symmetry", a quantum-dot analog of hidden symmetries in the quantum Hall effect (QHE) [1, 2]. As pointed out by MacDonald and Rezayi [1] and Chen and Quinn [1] this hidden symmetry can be understood by a mapping of the electron-hole system into a spin up/spin down electron system. The symmetric interactions translate into the spin-independent electron-electron interactions. The hidden symmetry is equivalent to the total spin being a con- 
served quantity. Due to Zeeman energy, in 2D systems in a strong magnetic field one can neglect electron spin. Since quantum dots emulate high magnetic field effects in terms of orbital degeneracies only, spin cannot be neglected. Spin starts playing a role when we have more than one carrier of the same type. This has been anticipated by constructing another relevant operator $Q^{+}$creating singlet bi-excitons: $Q^{+}=(1 / 2) \sum_{i, j}\left(c_{i, \downarrow}^{+} c_{j, \uparrow}^{+}+c_{j, \downarrow}^{+} c_{i, \uparrow}^{+}\right)\left(h_{i, \uparrow}^{+} h_{j, \downarrow}^{+}+h_{j, \uparrow}^{+} h_{i, \downarrow}^{+}\right)[2,18]$. The singlet operator involves the creation of pairs of electrons and pairs of valence holes with a total spin $S=0$, a much more complex object. Rather surprisingly, this bi-exciton operator was shown to satisfy a similar commutation relation as the polarization operator $\left[H, Q^{+}\right]=E_{X X} Q^{+}$, with $E_{X X}=2 E_{X}$. Hence, the application of $Q^{+}$to the vacuum generates a coherent state of singlet bi-excitons, and the energy of this bi-exciton state is twice the energy of a single exciton. The energy of the multiplicative state $\left(P^{+}\right)^{2}|v\rangle$ and the energy of the bi-exciton state $Q^{+}|v\rangle$ are degenerate. However, any small perturbation lowers the energy of the singlet-singlet state with respect to the triplet-triplet state. The bi-exciton operator involves pairing of electrons and pairing of holes, and plays an important role in determining the ground state of the interacting electron-hole system.

The presence of hidden symmetries implies that photons can only probe multiplicative states of the electron-hole system. One would however like to learn about other states, for example, what are the excitations of the electron system without the hole present, or what are the excited states of an electron-hole droplet. As we will show, this becomes possible when we introduce lateral confinement in the plane of the 2DEG.

\section{Single particle levels}

For a parabolic confinement appropriate for self-assembled quantum dots in a strong magnetic field, the spectrum of an electron (hole) is given by the Fock-Darwin spectrum of a single harmonic oscillator: $E_{m \sigma}^{\beta}=\Omega^{\beta}\left(m+\frac{1}{2}\right)$, with $m=0,1,2 \ldots, \sigma= \pm \frac{1}{2}, \Omega_{ \pm}^{\beta}=\Omega_{H}^{\beta} \pm \omega_{\mathrm{c}}^{\beta} / 2, \omega_{\mathrm{c}}^{\beta}=e B / m_{\beta}^{*} c$ (the cyclotron frequency for particle $\beta=$ electron or hole) and the hybridized frequency defined by $\Omega_{H}^{\beta}=$ $\sqrt{\omega_{\beta}^{2}+\left(\omega_{\mathrm{c}}^{\beta} / 2\right)^{2}}$. With the linear dispersion of single particle levels, the degeneracy of the Landau level is removed and the hidden symmetry no longer operates. The optical processes of spin polarized electrons in quantum dots described by the single harmonic oscillator spectrum and large Zeeman energy in the regime of filling factor one were described in detail in Ref. [16]. Recent experiments [14] indicate that the filling factor one will be difficult to achieve in available magnetic fields but the filling factor two should be achievable. The same is true for optically injected electron and hole systems where the filling factor two droplet was already realized [14]. Hence we present preliminary results of calculations for electron and exciton droplets at the filling factor two. 


\section{Excitonic quantum Hall droplets}

We first discuss the emission spectrum of the excitonic droplet. Examples of initial configurations of the two possible droplets, with an even number of excitons $N x=2 N$ and with an odd number of excitons $N x=2 N+1$, are shown in Fig. 1a. The emission from all occupied electron/hole levels is possible. The removal of an exciton from the odd-exciton droplet leaves an excited even-exciton droplet. Hence one needs to understand the excitation spectrum of the even-electron droplet. Figure 1b shows the ground and lowest excited states of the even-exciton droplet with $N x=2 N$. The excited states can be classified by the total spin of the electron and of the hole system. The singlet-singlet $(S / S)$ configurations include

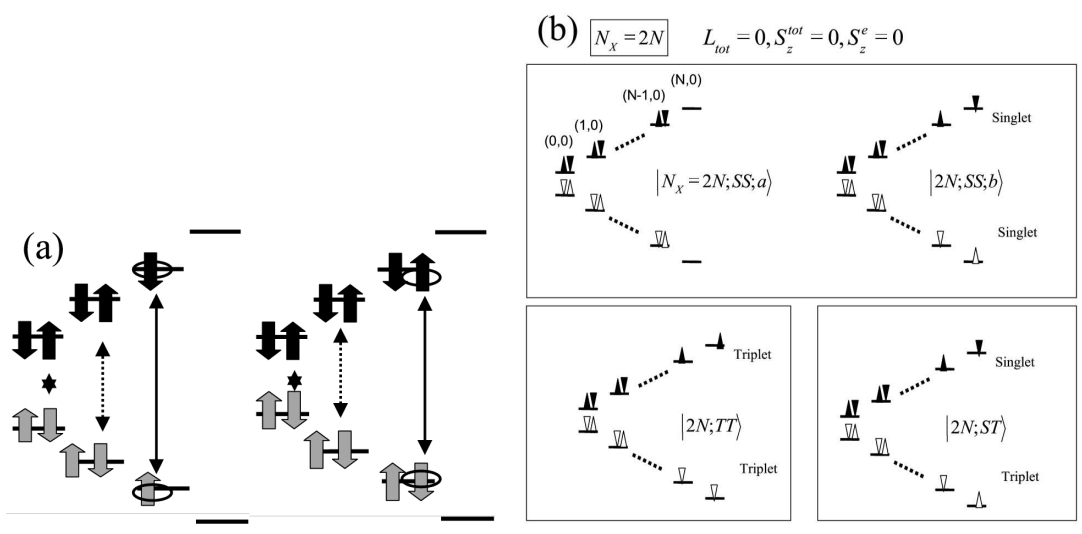

Fig. 1. (a) Example of exciton droplet in a quantum dot at the filling factor 2 with odd and even exciton numbers. Possible optical transitions are indicated by arrows. (b) Examples of the low energy excited states of the droplet classified by spin of electrons and of holes.

the ground state $|N x=2 N, S S, a\rangle$ and excited state $|N x=2 N, S S, b\rangle$. One can add a number of such one-pair excitations equal to the number of occupied electron-hole levels, but also multi-pair excitations to this group. In addition to the singlet-singlet excited configurations, there are singlet-triplet, triplet-singlet, and triplet-triplet configurations. One can construct all configurations with a given spin, diagonalize the Hamiltonian, and calculate the emission spectrum, as discussed in Ref. [20]. For illustrative purposes we chose parameters corresponding to hidden symmetry in the absence of confinement, i.e. we treat holes on the same level as electrons. The result for the $N x=5$ excitonic droplet is shown in Fig. 2a. The highest energy transition corresponds to the removal of the electron-hole pair at the Fermi level, leaving the even exciton droplet in the ground state. The next group of peaks consists of three peaks: the lowest in energy is the triplet/triplet excited state, the second one is the singlet/triplet combination, and the third state, with the lowest oscillator strength, is the singlet/singlet excited state. Hence the 

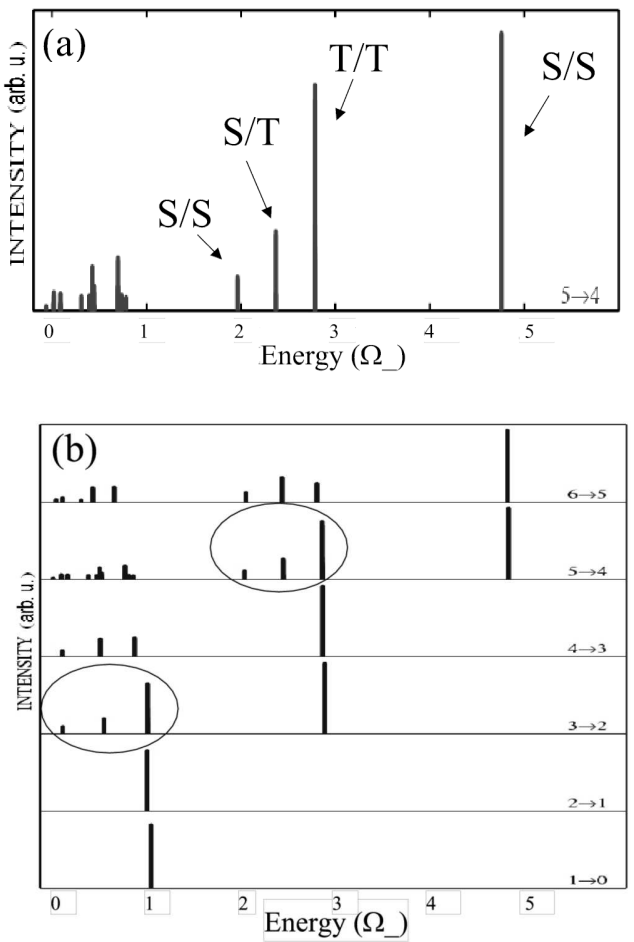

Fig. 2. (a) Emission spectrum intensity as a function of photon energy of $N x=5$ exciton droplet. The states shown in Fig. 1b are indicated. (b) Emission spectra for increasing number of excitons in filling factor 2 droplets. Characteristic emission spectra of odd exciton droplets are circled out.

emission spectrum directly reflects the excitation spectrum of the $N x=4$ droplet. The calculated evolution of the emission spectrum with an increasing number of excitons, shown in Fig. 2b, shows that the characteristic structure of the emission from the odd exciton droplets repeats itself. The relevant spectra are indicated by circles. The similar analysis can be applied to the emission from even exciton droplets, which are included in Fig. 2b. Hence, the calculations show that the emission spectra from excitonic droplets reveal the characteristic spin dependent structure of the excitation spectrum. The spectra are dominated by the emission to the ground and low lying excited states.

\section{Excitonic quantum Hall droplets}

Let us now turn to the emission from the electronic droplet, where the situation is different. Our objective is to infer electronic excitations of the droplet from the emission spectrum. An example of the $N e=6$ configuration at the filling factor $\nu=2[20,21]$ is shown in Fig. 3a. It shows $N e=6$ electrons occupying the 


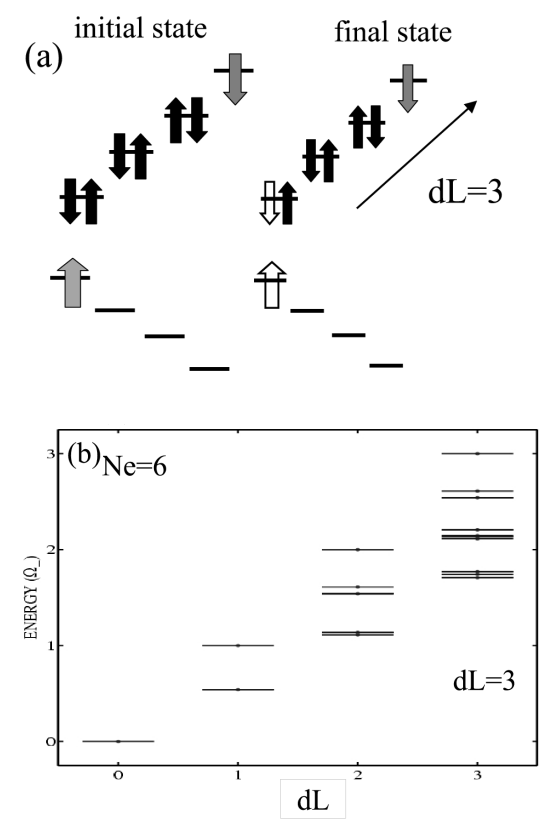

Fig. 3. (a) Initial configuration of $N e=6$ electron droplet plus one photo-excited electron-hole pair and final state configuration-excitation of $N e=6$ droplet at the filling factor 2 with the angular momentum $d L=N e / 2$. (b) Excitation spectra of $N e=6$ droplet as a function of angular momentum $d L$.

lowest spin degenerate orbitals $m=0,1,2$. The photo-excited electron occupies the first empty orbital $m=3$ while the valence hole is relaxed to the top of the valence band at $m=0$. There are of course more configurations which contribute to the ground state but this is the dominant one. The final state after the emission of a photon is shown in the right top panel of Fig. 3a. This state consists of a hole (missing electron) at $m=0$ and one extra electron at $m=3$ in the $\nu=2$ droplet. Hence the optically created final state is a one-pair excitation with an angular momentum $d L=N e / 2(d L=3)$. There are more excitations in the Hilbert space defined by a total angular momentum $d L=N e / 2$. In our example, the total number of excitations is ten. In Fig. 3b we show the excitation spectrum of the $\nu=2$ droplet as a function of $d L$. There are two states with $d L=1$, five with $d L=2$, and ten with $d L=3$, and so on. The highest energy state in each $d L$ Hilbert space corresponds to the center of mass excitations and has the energy $E=\Omega_{-}^{\mathrm{e}} d L$, independent of the number of electrons and electron-electron interactions. Of this excitation spectrum, only the part corresponding to $d L=N e / 2$ is accessible optically. In Fig. 4 we show the excitation spectrum classified by total spin (the two lower panels) and the emission spectrum. The excitation spectrum consists of 5 triplet $(S=1)$ and five singlet $(S=0)$ states, with the energy of excitations counted to the left, i.e. the lowest excited states correspond to the 


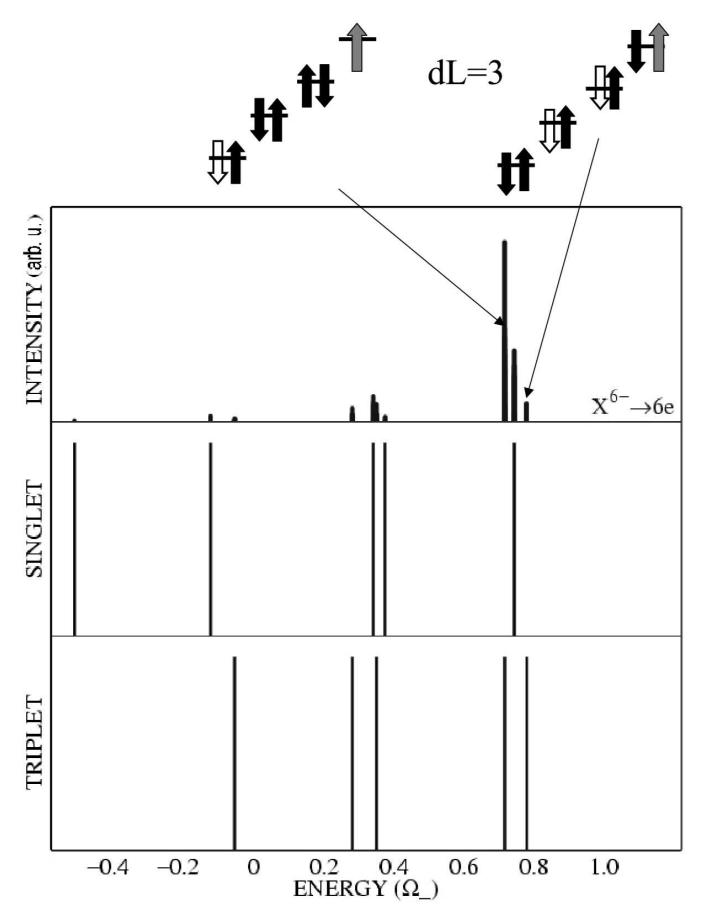

Fig. 4. Excitation spectra of $N e=6$ droplet for angular momentum $d L=3$ classified into triplet $(S=1)$ and singlet $(S=0)$ branches and corresponding emission spectrum. Dominant configurations corresponding to low energy spin triplet excitations are indicated. Note the internal spin flip bi-exciton configuration as the lowest state observable in emission.

highest energy emission peaks. The highest energy emission peaks correspond to the three lowest energy excited states, two triplets and one singlet. The lowest energy triplet, illustrated by a dominant configuration, is shown in Fig. 4 . It corresponds to two pairs of excitations, i.e. there are two holes in the $\nu=2$ droplet and two spin polarized electrons. This is a correlated configuration corresponding to a spin flip bi-exciton. This dark configuration is coupled to an optically active one pair excitation, also shown in Fig. 4, and hence visible in the emission spectrum. All configurations are visible in the emission spectrum, with oscillator strength larger at a higher energy. The characteristic pattern of the emission spectra as a function of the number of electrons is shown in Fig. 5. The emission from the exciton is followed by the emission from the charged exciton, shifted to a lower energy, followed by increasing red shifted emission from highly charged excitons. The characteristic pattern of the emission reflects the characteristic pattern of the excitation spectrum of the $\nu=2$ droplet, shown in the lower panel. We see that the emission from $X+2 e$ has two peaks as it corresponds to $d L=1$, the emission from $X+4 e$ has 5 peaks as it corresponds to $d L=2$, and emission from $X+6 e$ 

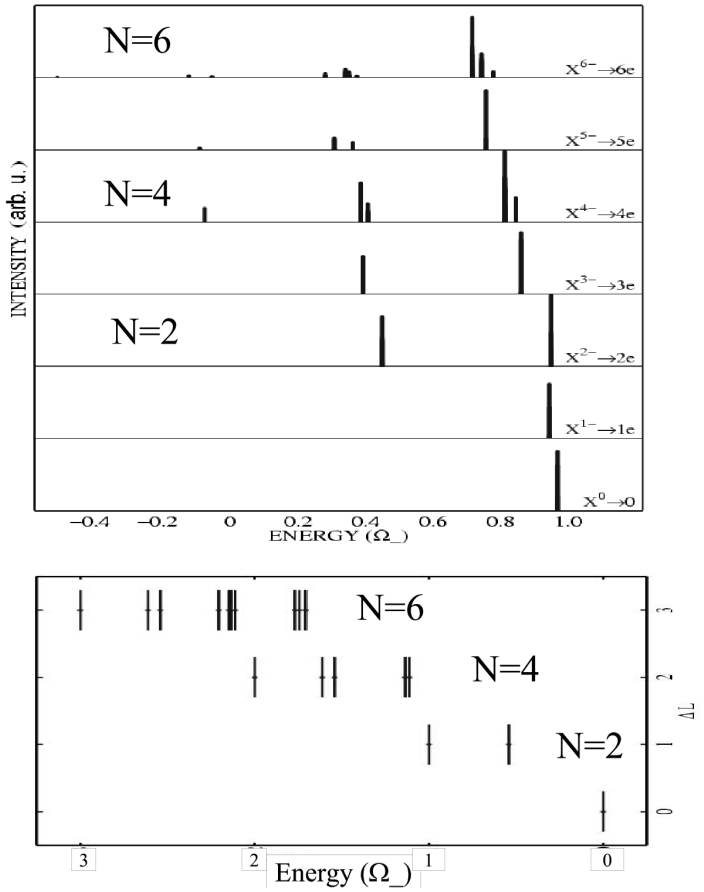

Fig. 5. Top panel: emission spectra for increasing number of electrons in the filling factor two electronic droplet. Note the characteristic structure of low energy emission line for even electron final state. Bottom panel: excitation spectra of the filling factor 2 droplet, for comparison with the emission spectra.

has 10 peaks as it corresponds to $d L=3$. The energies are renormalized but the unique structure of the entire excitation spectrum of the $\nu=2$ droplet can be extracted from emission experiments.

\section{Conclusions}

In summary, we presented a method to extract excitation spectra of a prototype chiral Fermi droplet ( $\nu=2$ droplet) of interacting electrons and/or excitons from interband emission spectra.

\section{Acknowledgment}

The authors thank M. Korkusinski, M. Potemski, and S. Raymond for discussions. 


\section{References}

[1] I.V. Lerner, Yu.E. Lozovik, Zh. Eksp. Teor. Fiz. 80, 1488 (1981) [Sov. Phys. JETP 53, 763 (1981)]; D. Paquet, T.M. Rice, K. Ueda, Phys. Rev. B 32, 5208 (1985); A.H. MacDonald, E.H. Rezayi, Phys. Rev. B 42, 3224 (1990); Yu.A. Bychkov, E.I. Rashba, Phys. Rev. B 44, 6212 (1991); X.M. Chen, J.J. Quinn, Phys. Rev. Lett. 70, 2130 (1993); V. Apalkov, E. Rashba, Phys. Rev. B 46, 1628 (1992).

[2] For recent review, see P. Hawrylak, Solid State Commun. 127, 753 (2003).

[3] A. Wojs, P. Hawrylak, Phys. Rev. B 51, 10, 880 (1995).

[4] J.J. Palacios, D. Yoshioka, A.H. MacDonald, Phys. Rev. B 54, R2296 (1996).

[5] A.B. Dzyubenko, A.Yu. Sivachenko, Phys. Rev. Lett. 84, 4429 (2000).

[6] A. Wojs, P. Hawrylak, J.J. Quinn, Phys. Rev. B 60, 11661 (1999); A. Wojs, J.J. Quinn, P. Hawrylak, Phys. Rev. B 62, 4630 (2000).

[7] G. Finkelstein, H. Shtrikman, I. Bar-Joseph, Phys. Rev. B 56, 10326 (1997).

[8] A.J. Turberfield, S.R. Haynes, P.A. Wright, R.A. Ford, R.G. Clark, J.F. Ryan, J.J. Harris, C.T. Foxon, Phys. Rev. Lett. 65, 637 (1990).

[9] N.J. Pulsford, I.V. Kukushkin, P. Hawrylak, K. Ploog, R.J. Haug, K. von Klitzing, V.B. Timofeev, Phys. Status Solidi B 173, 271 (1992).

[10] Z.X. Jiang, B.D. McCombe, P. Hawrylak, Phys. Rev. Lett. 80, 3344 (1998).

[11] S. Patel, A.S .Plaut, P. Hawrylak, H. Lage, P. Grambow, D. Heitmann, K. von Klitzing, J.P. Harbison, L.T. Florez, Solid State Commun. 101, 865 (1997).

[12] H. Drexler, D. Leonard, W. Hansen, J.P. Kotthaus, P.M. Petroff, Phys. Rev. Lett. 73, 2252 (1994).

[13] K. Karrai, R.J. Warburton, Ch. Schulhauser, A. Högele, B. Urbaszek, E.J. McGhee, A.O. Govorov, J.M. Garcia, B.D. Gerardot, P.M. Petroff, Nature 427, 136 (2004).

[14] S. Raymond, S. Studenikin, A. Sachrajda, Z. Wasilewski, S.J. Cheng, W. Sheng, P. Hawrylak, A. Babinski, M. Potemski, G. Ortner, M. Bayer, Phys. Rev. Lett. 92, 187402 (2004).

[15] P. Hawrylak, D. Pfannkuche, Phys. Rev. Lett. 70, 485 (1993).

[16] P. Hawrylak, A. Wojs, J.A. Brum, Phys. Rev. B 54, 11397 (1996).

[17] A. Wojs, P. Hawrylak, Phys. Rev. B 55, 13066 (1997).

[18] G.A. Narvaez, P. Hawrylak, Phys. Rev. B 61, 13753 (2000).

[19] A. Wojs, P. Hawrylak, Solid State Commun. 100, 487 (1996); P. Hawrylak, Phys. Rev. B 60, 5597 (1999).

[20] S.J. Cheng, W. Sheng, P. Hawrylak, Phys. Rev. B 68, 235330 (2003).

[21] A. Wensauer, M. Korkusinski, P. Hawrylak, Phys. Rev. B 67, 035325 (2003); M. Ciorga, A. Wensauer, M. Pioro-Ladriere, M. Korkusinski, J. Kyriakidis, A.S. Sachrajda, P. Hawrylak, Phys. Rev. Lett. 88, 256804 (2002). 